\title{
UNA FLAGELACIÓN DE JOAN REIXACH DE COLECCIÓN PARTICULAR. NUEVOS DOCUMENTOS Y CONSIDERACIONES SOBRE EL BINOMIO JACOMART-REIXACH ${ }^{1}$
}

\author{
Ximo Company, Borja Franco, Isidro Puig, Joan Aliaga y Stefania Rusconi
}

\begin{abstract}
La atribución a Joan Reixach de una tabla con la Flagelación, nos ha permitido aportar noticias inéditas en torno a su autor y a los lazos profesionales que pudieron unirle en ocasiones con Jacomart. Una asociación tácita demostrada documentalmente con una nueva noticia fechada en 1449 que explicaría la proximidad estilística y contractual de algunas de sus obras.

Palabras clave: Pintura valenciana; siglo XV; Reixach; Jacomart.

\section{A FLAGELLATION BY JOAN REIXACH IN A PRIVATE COLLECTION: NEW DOCUMENTS AND CONSIDERATIONS ON THE COLLABORATION JACOMART-REIXACH}

The attribution to Joan Reixach of a panel representing the Flagellation, has allowed us to contribute unpublished news concerning its author and his occasional professional links with Jacomart. This tacit relationship is now demonstrated by a new document of 1449 , which would explain the contractual and stylistic proximity of some of their works.

Key words: Valencian painting; $15^{\text {th }}$ century; Reixach; Jacomart.

La catalogación y análisis de una pieza casi desconocida ${ }^{2}$ siempre conlleva la revisión y el estudio en torno a sus artífices. Este trabajo nos sirve como acicate para continuar profundizando en el conocimiento de las fuentes documentales e historiográficas, localizar contratos de obras que nos lleven a reubicar otras tablas dispersas que pudieran emparentarse con la que hemos analizado y completar el extenso corpus artístico de Joan Reixach, uno de los máximos representantes de la renovación artística valenciana de mediados del siglo XV. En este caso en concreto, nuestro interés se centró en un óleo y temple sobre tabla de pino, conservado en la colección particular Laia Bosch. En él vemos representada la Flagelación de Cristo (fig. 1) y, por las razones que expondremos a continuación, su cronología deberíamos situarla aproximadamente hacia 1455, si bien desconocemos su origen y creemos que pertenecería a un conjunto mayor, seguramente a la predela de un retablo dedicado a la Pasión de Cristo. A día de hoy no hemos podido encontrar ninguna otra tabla de este pintor, al menos conocida, que pudiera completar toda la secuencia, aunque planteamos la hipótesis de que perteneciera a una predela basándonos en las semejanzas que se observan con otras composiciones similares del mismo autor, como son la predela de la Pasión del Museo de Bellas Artes de Valencia (inv. 2101, fig. 2), la Flagelación (inv. 208, fig. 3) de mayores dimensiones $(111 \times 130 \mathrm{~cm})$ que procedería del banco del retablo dedicado a los Siete Gozos de la Virgen del Monasterio de Portaceli (Serra, Valencia, c. 14601470), en la actualidad también en el museo anteriormente citado.

Uno de los aspectos más importantes de la obra de Reixach es su conocida unidad estilística. Partiendo de la tradición del gótico internacional, fue incluyendo distintos elementos que lo acercan a las nuevas corrientes flamenquizantes, sin perder, en ningún momento, sus señas de identidad propias, muchas veces marcadas por el peculiar decorativismo de su región de origen. Es habitual en él, por ejemplo, el uso de azulejos con distintas formas y coloraciones, conocidos como "escarabadets". Valencia, y en especial Manises, desarrolló en aquel momento un gran

\footnotetext{
${ }^{1}$ Este artículo se incluye dentro del trabajo del Proyecto de Investigación I+D+I del Ministerio de Ciencia e Innovación titulado: La configuración de la Pintura Mediterránea del primer Renacimiento en la Corona de Aragón (c.14401525). Problemas de pintura (HAR2009-07740; en el que participan los autores de este artículo, Universitat de Lleida). Así como del Grup de Recerca Consolidat reconocido por el Departament d'Innovació, Universitats i Empresa de la Generalitat de Catalunya: “Art i Cultura d’Època Moderna” (ACEM) de la Universitat de Lleida (2009 SGR 348).

${ }^{2}$ Esa pieza fue estudiada de modo breve y preliminar en: CompanY-Puig, 2007: 330-333.
} 


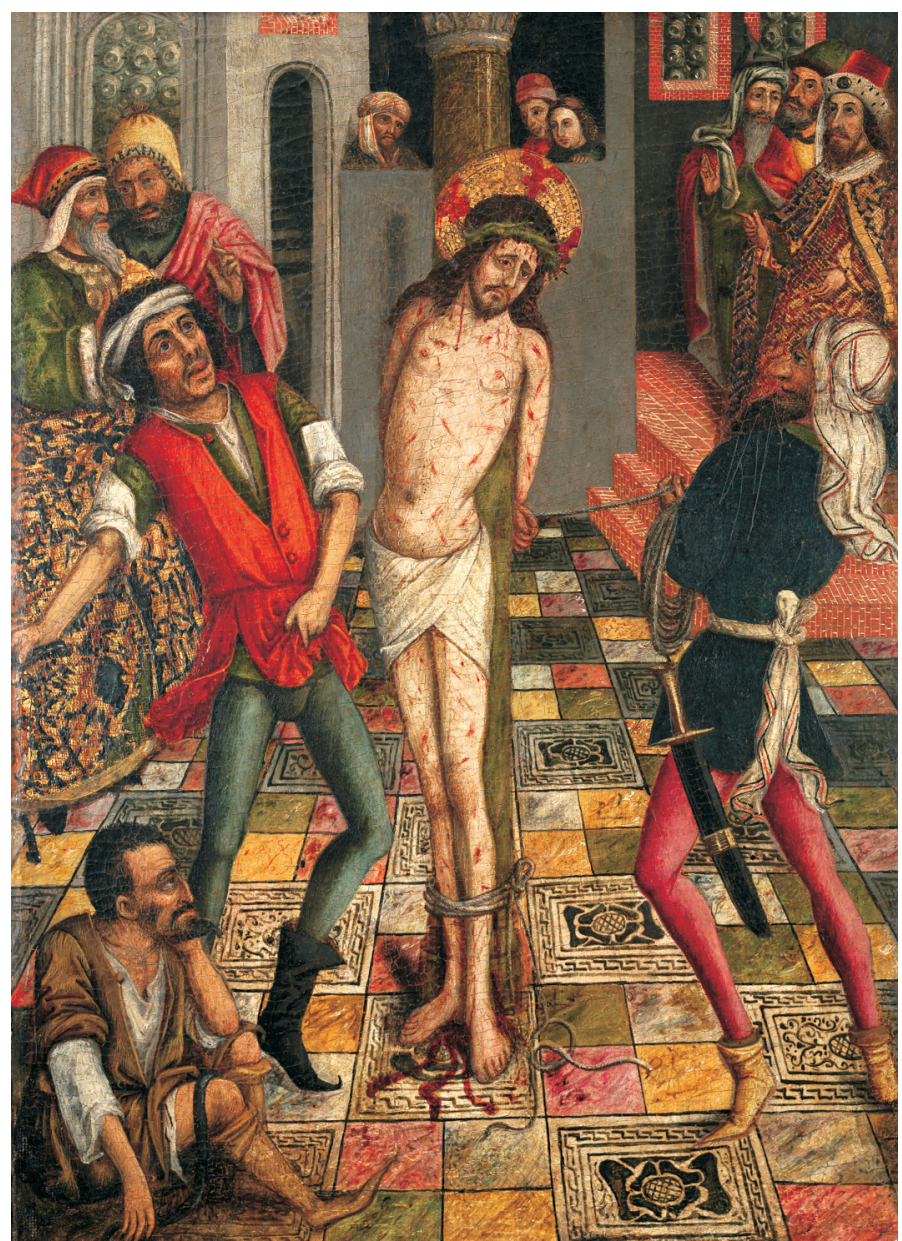

Fig. 1. Joan Reixach, Flagelación de Cristo, h. 1455, colección Laia Bosch.

comercio de este tipo de producto manufacturado que, a través del creciente puerto de Valencia, se exportaba al exterior. Así pues, Reixach, deudor del ambiente en el que nació, decidió eliminar los monócromos suelos de sus interiores y decorarlos al estilo de los palacetes medievales valencianos. Este tipo de alicatado que apreciamos en la tabla de la colección Laia-Bosch, lo encontramos también, por ejemplo, en una de sus pocas obras firmadas, el Retablo de Santa Úrsula, conservado en el MNAC de Barcelona, que procedería del mismo periodo que la tabla que analizamos. Del mismo modo aparece en el Retablo de San Lorenzo y San Pedro de Verona de Catí (fig. 4), obra contratada en 1460 por Jacomart, aunque con segura participación del taller de Reixach.

También repite algunos elementos arquitectónicos como una suerte de medias esferas en los ventanales que representa de fondo en sus tablas y que podemos encontrar en la escena del martirio de Santa Catalina del Retablo de la Iglesia de la Natividad de Nuestra Señora de Villahermosa del Río (Castellón), así como en la predicación de San Pedro de Verona en el anteriormente citado Retablo de San Lorenzo y San Pedro de Verona de Catí (fig. 4). De hecho, arquitectónicamente podemos encontrar una simbiosis entre un incipiente clasicismo y la todavía moda pictórica imperante del mundo gótico. Ciertamente, el interior de la escena nos remite más 


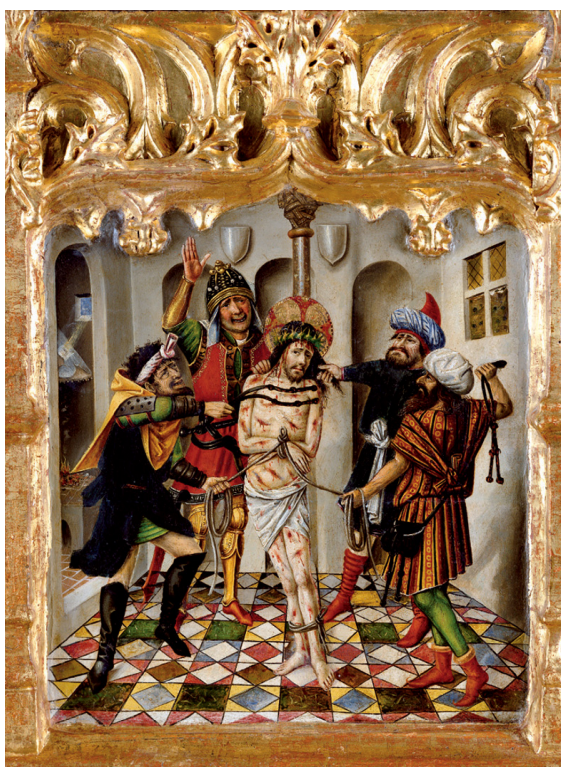

Fig. 2 Joan Reixach, Flagelación de Cristo de la predela de la Pasión, h. 1450, Museo de Bellas Artes de Valencia (inv. 2101).

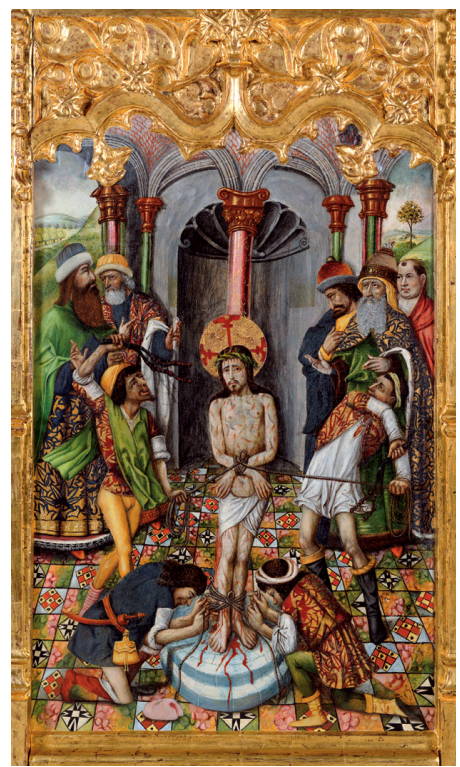

Fig. 3. Joan Reixach, Flagelación de Cristo de la predela de la Pasión del retablo dedicado a los Siete Gozos de la Virgen del Monasterio de Portaceli, h. 1460, Museo de Bellas Artes de Valencia (inv. 208).

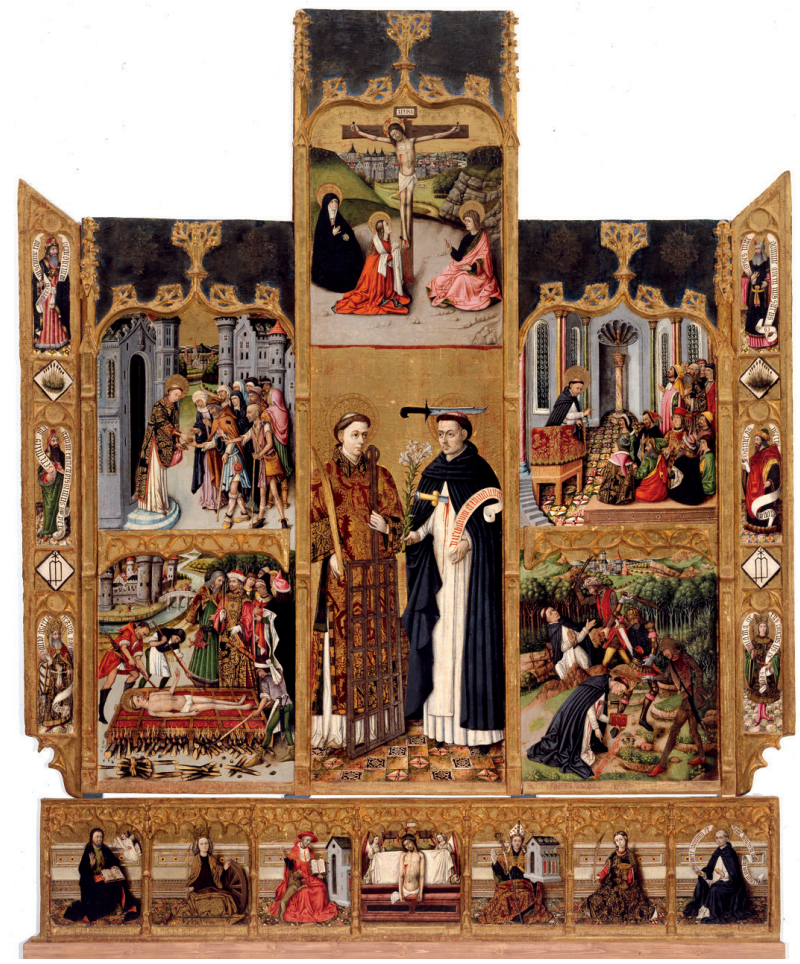

Fig. 4. Joan Reixach-Jacomart. Retablo de San Lorenzo y San Pedro de Verona, 1460, Iglesia Parroquial de Catí. 
hacia una arquitectura medieval que hacia un templo renacentista, pero el uso del arco de medio punto así como el pequeño fragmento de capitel que se deja entrever en la columna donde Cristo fue flagelado nos indican esa transición citada. De hecho, estos vanos así como el capitel se asemejan más al que realizara en la Flagelación de la Predela de la Pasión del Museo de Bellas Artes de Valencia (fig. 2) que a los que aparecen en esta misma escena de la predela homónima pintada unos años después (hacia 1460-1470) conservada en el museo valenciano (fig. 3) pero procedente de la Cartuja de Portaceli, con esa hornacina avenerada y unas nervaduras relacionadas con el mundo clásico. Este hecho nos serviría para datar la tabla que analizamos con una fecha anterior a 1465, porque, en este aspecto, muestra cierto arcaísmo respecto a las realizaciones citadas.

También Reixach repitió diversos tipos fisonómicos de sus personajes que nos permiten crear filiaciones entre sus obras. Hemos encontrado similitudes en el rostro del Salvador y su nimbo crucífero con las flagelaciones anteriormente citadas, así como una relación con otros aspectos anatómicos en diversas tablas de este mismo autor. Este hecho es palpable si analizamos el cuerpo de San Lorenzo, en la escena de su martirio dentro del Retablo de San Lorenzo y San Pedro de Verona de Catí (fig. 4). Se aprecia claramente cómo la forma de resolver el cuerpo desnudo, así como la forma de los pectorales del Cristo son casi idénticos a los del santo citado.

Por otro lado, el estudio de la obra con la técnica de la fotografía digital de infrarrojos nos ha permitido comprobar cómo el pintor ha utilizado diferentes instrumentos de trabajo y materiales para elaborar su composición (fig. 5). Las zonas donde utiliza la incisión con punzón son

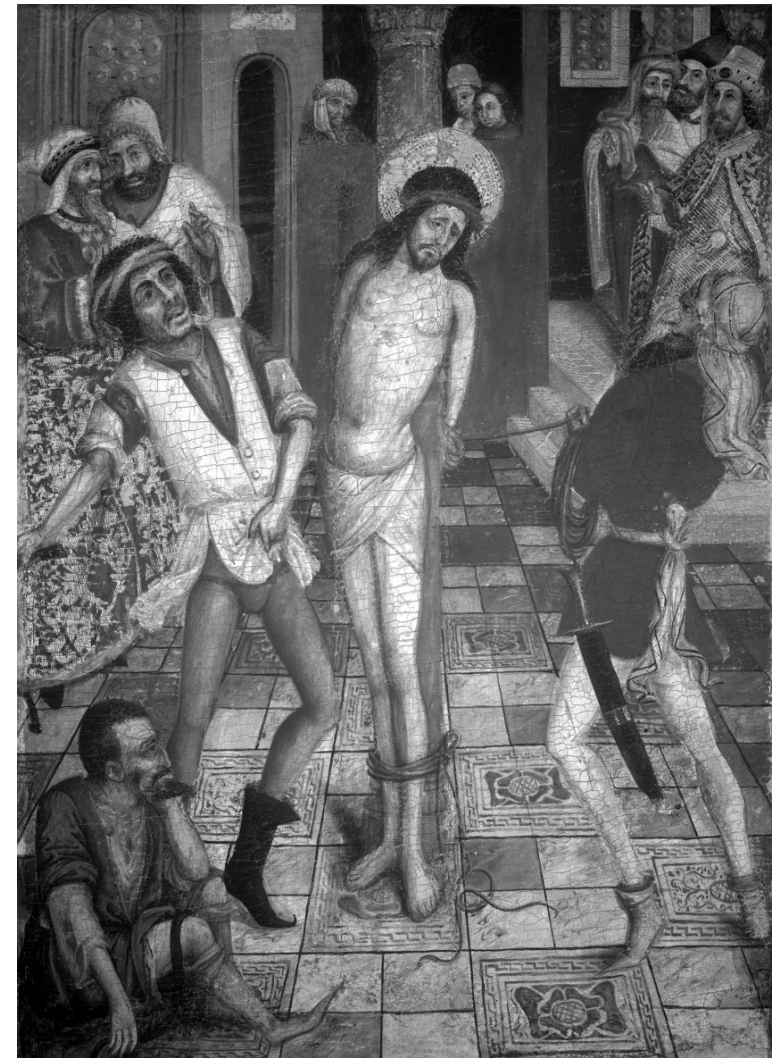

Fig. 5. Reflectografía de infrarrojos de la Flagelación de Cristo, colección Laia Bosch. 
las que delimitan los nimbos y los vestidos con brocados, en los que se aplica el oro; superficies áuricas que después decora con pequeñas incisiones o leves burilados de forma circular. Todo ello se observa en los ropajes de dos de los personajes, uno situado a la izquierda de la composición y el otro en el ángulo superior derecho de la tabla. También apreciamos las mismas incisiones en el sayón que sujeta a Cristo con la soga, lo cual nos hace intuir que en un primer momento el artista tenía la intención de aplicar la decoración de brocado en su túnica, lo que finalmente desestimó, quizá porque la condición social de un esbirro no es compatible con la riqueza, distinción y elegancia que presupone a los personajes que lucen este tipo de brocados. Normalmente los trazos con líneas incisas realizada con un estilete también se utilizan para marcar las perspectivas. Sin embargo, en el caso de la tabla que nos ocupa, Reixach olvida el estilete y prefiere señalar la perspectiva del solado con unas líneas directamente realizadas a pincel, a base de un medio fluido diluido, todo a mano alzada, como advertimos en los azulejos de mayor tamaño. Finalmente Reixach y otros miembros de su taller repasan todas las líneas geométricas con un trazo negro algo más pronunciado e insistido. En el resto de la composición todo se delimita con pinceladas suaves y ligeros trazos, que bien podríamos considerar, a la postre, como una especie de dibujo subyacente. Con él marca algunas sombras, pliegues de los ropajes y contornos de las figuras, cuyos perfiles, en la aplicación definitiva de la policromía, repasa con líneas de tonos de color más oscuros.

Con todo lo dicho Reixach exhibe una preocupación hasta ahora bastante discreta por el tema del dibujo a carboncillo tan típico en la época. Él prefiere mitigar, diluir o incluso confundir los perfiles básicos de sus figuras, o bien dejarlos entrever con puras líneas pictóricas de buen impacto lumínico, tal como se aprecia, por ejemplo, en el paño de pureza de Cristo, en su torso o en los perfiles de las piernas de esta misma figura.

Una vez analizada la obra y presentados todos aquellos indicios que nos incitan a atribuirla con toda seguridad a Reixach, haremos un breve recorrido por algunos aspectos biográficos inéditos, fruto de nuestra reciente investigación archivística.

Hijo del escultor catalán Llorenç Reixac, "obrerius ymaginarum, civis Barchinone"3, no sabemos a ciencia cierta la fecha de su nacimiento, aunque consideramos que probablemente tendría lugar en Barcelona en torno a 14114, dado que el 13 de diciembre 1431 ya aparece por pri-

\footnotetext{
${ }^{3}$ Madurell Marión, 1945: 310. El hecho de la ciudadanía barcelonesa de Llorenç Reixach, permite abrigar la hipótesis de que su hijo Joan hubiera podido nacer en Barcelona, aunque nada podemos asegurar con plena certeza. Sobre LLORENÇ REIXACH cfr. TERÉS, 1987: 171-185.

4 En favor del hipotético nacimiento de Joan Reixach en Barcelona, debemos recordar que el 13 de marzo de 1447 éste aún tenía actividades, bienes y propiedades en Barcelona, como lo demuestra el hecho de que nombrara procurador a su padre Llorenç, para que lo representara en todos sus asuntos en Barcelona y el Principado de Cataluña, véase: Protocol Bertomeu Tovià, el menor, núm. 25.047, Archivo de Protocolos de Valencia, (APPV), publicado por CERVERó Gomis, 1966: 29. Pero sabemos, además, que el 2 de mayo de 1447, Joan Reixach aparece como heredero universal de su difunta madre Constanza, que vivió hasta su muerte en Barcelona, y de nuevo nombra procurador a su padre Llorenç Reixach para que pueda vender al barcelonés Miquel Ferrando, "pellipario", algunas posesiones que Joan Reixach había heredado de su madre en el barrio "lo comple" de Barcelona: "Iohannes Rexach, pictor civis civitatis Valencie, filius et heres universalis dompne Constancie, quondam, matris mee, que fuit uxor vestri Laurencii Rexach ymaginarii civis Barchinone, patris mei ab iurestato defuncte scienter et gratis facio constituo et ordino procuratorem meum certum et specialem et in subscriptis generalem vos dictum Laurencium Rexach, genitorem meum, licet absentem tanquam presentem, videlicet ad laudandum ac probandum, retificandum et confirmandum pro me et nomine meo quandam vendicionem per vos precio septuaginta librarum Barchinone factam Miquaeli Ferrando pellipario, civi dicte civitatis Barchinone, de totis illis domibus cum tabulis et cum intratibus, exitibus, iuribus et pertinenciis earum quas habebatis et possidebatis in iam dicta civitate Barchinone in vico vocato "lo comple"' (Protocols de Bertomeu Tovià, APPV, núm. 25.047 , doc. Inédito,). Queda muy claro, en cualquier caso, que son muy evidentes las vinculaciones de Joan Reixach con la ciudad de Barcelona.
} 


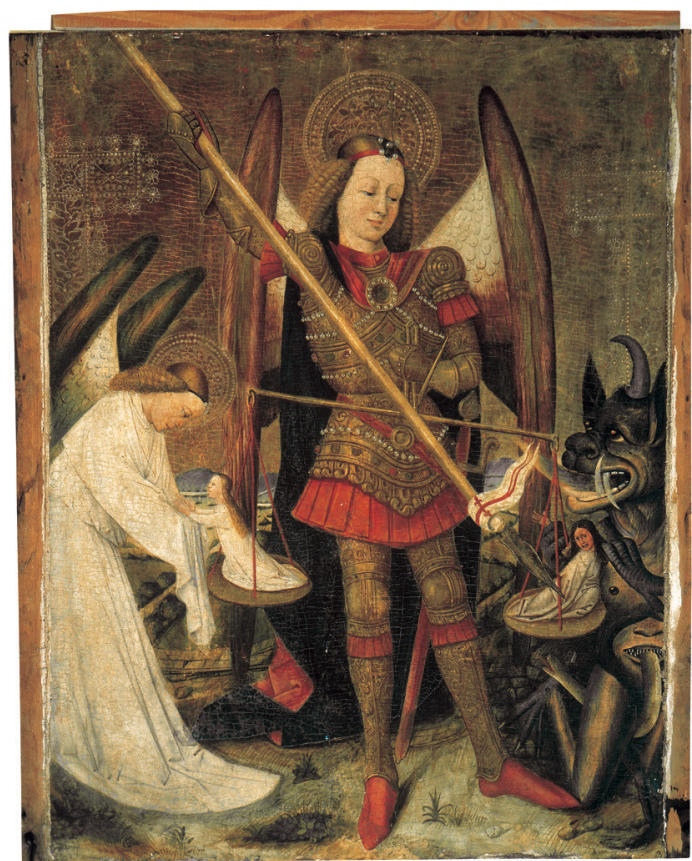

Fig. 6. Jacomart (?). San Miguel pesando las almas, h. 1441, Galleria Parmeggiani de Regio Emilia, procedente de la Iglesia Parroquial de Burjassot (Valencia). mera vez documentado como mayor de edad trabajando en Zaragoza ${ }^{5}$. Poco conocemos de sus trabajos iniciales, salvo algunas referencias documentales de obras a día de hoy perdidas, como el Retablo de la advocación de los Siete Gozos de María, destinado a una capilla de una huerta cercana al desaparecido Palau Reial de Valencia (1437-1440), aunque sabemos que este retablo acabaría yendo a parar a la capilla del Castillo de Xàtiva (14391440).

El 5 de febrero de 1438 fue convocado para la tasación de una obra del maestro Lluís Dalmau $^{6}$, iniciándose su primera gran etapa productiva con la realización de diversas pinturas para la cartuja de Portaceli (1439-1440), el Retablo de Santa Bárbara para la Iglesia de San Juan del Hospital de Valencia (1440-1441) u obras diversas para la Catedral de Valencia. También procede de este momento el encargo de continuar el Retablo de San Miguel de Burjassot (fig. 6), que había iniciado Jacomart en 1441 y había dejado inacabado, después de haber concluido "una imatge de sent Miquel e altres obres"7. Es muy importante aclarar que en Burjassot Jacomart no pintó únicamente una tabla de San Miguel como apuntó Benito en 2001: "sólo tenía hecha la tabla central".

Hemos revisado la documentación alusiva al contrato de Jacomart en Burjassot ( 28 de febrero de 1441) y en todas las referencias a lo realizado por este pintor en dicho retablo se especifica que realizó, no una, la central, sino varias tablas más: "en lo qual dit retaule lo dit Jacomart havia ja pintat una imatge de sent Miquel e altres obres"9. Por todo ello, y la apreciación no es para nada baladí, consideramos que el San Miguel pesando las almas de la Galleria Parmeggiani de Reggio Emilia, que procede de Burjassot, pudiera ser, con bastante verosimilitud, una obra inequívoca de Jacomart, lo que vertiría mucha luz sobre la definitiva comprensión de su controvertida figura y de su verdadera y muy debatida producción pictórica.

Así pues, podríamos estar de acuerdo con los estudios de Josep Ferre Puerto donde comenta que la mencionada tabla de Reggio, por su temática y medidas, $92 \times 70 \mathrm{~cm}$, pudiera ser una tabla

\footnotetext{
5 SERRANO, 1916: 469.

${ }^{6}$ Aunque Sanchis Sivera y otros autores dieron fecha del 5 de enero de 1439, nuestra revisión en el Arxiu del Regne de València (ARV) realizada en 2010 certifica con claridad que se trata del 5 de febrero de 1438.

7 Registre d'àpoques de la Batlia, ARV, núm. 46, f. 323; documentación que hemos revisado, aunque publicada por TORMO, 1913: 107.

8 Benito Doménech, 2001: 37.

9 Registre d'àpoques de la Batlia, ARV, núm. 46, f. 323. Esta afirmación viene completada por otros textos sobre dicha obra, que ratifican nuestra postura: "per rahó de la imatge de Sent Miquel per lo dit mestre Jacomart feta e pintada en la peça migana del dessús dit retaule de Sent Miquel e en altres obres de aquell [...] per rahó de un retaule lo qual havia emprés de fer e pintar, e és cert que per certes imatges, figures e obres, les quals lo dit mestre Jacomart havia fetes e obrades en lo dit retaule" (Mestre Racional, ARV, 58, f. 304v-305v.)
} 
lateral, como sucede en un Retablo de San Miguel del Museo Episcopal de Vic. Según este autor, la tabla de Burjassot estaría relacionada con esta obra de Vic, atribuida a un pintor muy cercano a Jacomart-Reixach, un tal Pere Girard o Maestro de Cervera. Sin embargo las medidas de la tabla central del retablo catalán son $113 \times 78 \mathrm{~cm}$, es decir apenas unos $10 \mathrm{~cm}$ mayor que la de Reggio, con lo que, al menos por medidas, la tabla de Reggio no debería ser obligatoriamente una tabla lateral sino que también podría ser alguna semicentral de Burjassot; la lateral de Vic mide $55 \times 50 \mathrm{~cm}$, con lo cual sería demasiado pequeña para relacionarla con la de Reggio de $92 \times 70 \mathrm{~cm}$. Por supuesto, estamos hablando de dos retablos diferentes. Queda claro, en cualquier caso, y este es el punto clave de toda esta larga disertación, que desde un punto de vista documental no existe ningún impedimento para refutar que la tabla de Reggio no pudiera ser la que Jacomart pintó en Burjassot, tanto si esta fuera una tabla lateral como una semicentral; en ambos casos la documentación exhumada permite mantener en toda regla la posible participación de Jacomart. A un nivel estilístico no podemos extendernos ahora en si el San Miguel de Reggio pudiera ser de Jacomart o de Reixach, pero sí procede señalar que pocos años después de trabajar Reixach en el retablo de Burjassot (1444-1445) realizó el Retablo de Santa Catalina de Alejandría, de Villahermosa del Río (Castellón) (pagada en 1448 pero con supuesto contrato de 1447), cuyos estilemas, marcadamente secos, muy dibujísticos y caligráficos, difieren bastante de los mucho más suaves y pictóricos exhibidos en el rostro del San Miguel de Reggio ${ }^{10}$.

Esta proximidad estilística entre Jacomart y Reixach se convirtió en una constante dentro de la trayectoria profesional del segundo. Es decir, algunos de los retablos que Reixach realizó hasta la muerte de Jacomart en 1461 fueron continuación de proyectos que, en principio, debiera haber ejecutado el mencionado Jacomart, hecho que ha permitido plantear que ambos hubieran podido colaborar en algunas de las obras por ellos contratadas. Cabe reconocer que actualmente existe una cierta confusión historiográfica entre la verdadera identidad de estos dos pintores, tal y como ya se ha comentado y seguiremos exponiendo más adelante. El hecho de que Reixach continuara algunas obras de Jacomart, así como las presumibles similitudes en su estilo, ha producido todo un aluvión de comentarios en torno a qué relación pudo haber entre uno y otro pintor. Cabe admitir que a fecha de hoy no están definitivamente resueltas las verdaderas personalidades de ambos pintores; de ahí que nos estemos extendiendo un poco en la exposición de esta compleja dualidad.

De hecho, si Reixach continuó en 1444 el retablo de Burjassot que Jacomart dejó inconcluso, parece lícito plantearse que uno y otro pintarían, cuando menos, de un modo similar; cabe establecer una razonable afinidad estilística entre ambos maestros. Es decir, no parece lógico, que se contrate a un pintor para realizar la continuación de una obra ya iniciada por otro maestro, cuyos estilemas, su forma de pintar y su sensibilidad creativa fueran muy distintos a los del primer pintor. Parece sensato pensar que siempre se buscaría a un segundo maestro lo más afín y parecido posible al primero. De otro modo las disonancias estilísticas hubieran podido ser perturbadoras a los ojos del promotor de la obra y a los ojos, por supuesto, de los fieles de la parroquia de Burjassot.

Otra curiosa coincidencia entre los dos maestros se produce cuando Jacomart contrató en 1450 un retablo para la iglesia de Museros, retablo que por razones que desconocemos se mantuvo en suspenso hasta que el 18 de noviembre de 1461, unos meses después de acaecida la muerte de Jacomart, Reixach de nuevo se hizo cargo del mismo con unas capitulaciones similares a las firmadas por Jacomart ${ }^{11}$.

Más adelante, en enero de 1460, Jacomart contrató el citado Retablo de San Lorenzo y San Pedro de Verona de la parroquial de Catí; debía entregarlo perfectamente acabado en el plazo de

10 Ferre Puerto, 1997: 311-320. Ferre Puerto, 2000: 1681-1686.

11 Gómez-FerRer, 1994: 20-24. GómEZ-Ferrer-CorbalÁn, 2003: 19-24. 
un año, y así pudo hacerse, pues Jacomart estuvo durante todo el mencionado año, y aún seis meses después, plenamente activo. Es cierto que lo contrató Jacomart, sin embargo, hoy nadie pone en duda que en sus definitivas hechuras formales, compositivas y coloristas, intervino Joan Reixach, muy probablemente trabajando junto a su compañero Jacomart, en un hipotético taller común de ambos.

Pero las coincidencias y la mencionada dualidad entre Jacomart y Reixach no acaban ahí. El 27 de junio de 1461 Jacomart, todavía plenamente activo, pintaba las rejas de la catedral de Valencia. De forma absolutamente repentina e inesperada moría apenas veinte días después, el 16 de julio. En menos de una semana Joan Reixach se había hecho cargo de la continuidad de la pintura de las rejas catedralicias ¿Por qué, nuevamente Reixach, y no otro pintor, y de forma tan inmediata que no le hizo falta ningún tipo de contrato? ¿No indica todo esto que quizá Jacomart y Reixach eran un sólo binomio, al menos a los ojos de los canónigos de la catedral?

La fortuna archivística aún nos ha permitido encontrar otros documentos en los que las relaciones entre Jacomart y Reixach se constatan de forma fehaciente. El 12 de agosto de 1462, fallecido ya Jacomart, su viuda Magdalena Devesa intervino en un pequeño asunto económico en el que pide a Jeroni Reixach, hijo de Joan Reixach, que actúe de testigo. Se trata de un documento muy escueto, pero esta vez nos sirve para comprobar que estamos ante dos familias que no sólo pudieron cooperar a un nivel profesional, sino que también actuaron con sólidos lazos de amistad o ayuda doméstica y familiar ${ }^{12}$.

Ciertamente, hasta ahora hemos estado hablando de datos documentales que en ningún caso vinculan de forma concluyente la colaboración profesional entre uno y otro pintor. Es decir, hasta ahora, cuando aparece Jacomart, Reixach no está, y viceversa; solamente los podíamos intuir juntos en la sombra. Sin embargo, ya hemos encontrado un documento en el que finalmente ambos maestros aparecen unidos; una referencia en la que leemos con claridad "magistros Iacobum Jacomart et Iohannem Rexach, pictores". Lleva fecha del 2 de febrero de 1449 y demuestra, ante todo, la fehaciente e indiscutible existencia de unos lazos profesionales entre ambos pintores, quienes actúan juntos en la proclamación de una sentencia arbitral que afecta al pintor valenciano Felipe Porta, quien cobra una cierta cantidad a cuenta de un retablo que realizó para la capilla lombarda del monasterio de San Francisco de Valencia ${ }^{13}$. No cabe duda que este importante hallazgo documental contribuye a perfilar un poco mejor las verdaderas personalidades de Jacomart y Joan Reixach. El nuevo documento nos exhorta, cuando menos, a ir más despacio y de forma mucho más reflexiva a la hora de historiar bien sus respectivas biografías y de reela-

12 Archivo de la Catedral de Valencia (ACV), protocolo núm. 3.680. También citado en: Sanchis Sivera, 1930: 170. Completado por nosotros, el documento dice lo siguiente: Magdalena, uxor in primis nubciis quondam honorabilis magistri Jacomart, alias Bacho, pictoris civis 'civitatis Valentie (...), firmavit apocam venerabili et discreto Berengario Company, presbitero domerio ac viceoperario fabrice Sedis Valentie, presenti, de centum quadraginta quatuor solidis regalium Valentie dicto viro suo debitis racione depingendi les faxes de ferre la una gran e l'altra xica cancelli sive rexat altaris maioris dicte sedis, unde renuncians et cetera. Testes inde sunt Petrus Trespo, cerdo, et Ieronimus Rexach, pictor, cives Valencie.

${ }^{13}$ Reproducimos a continuación este importante documento inédito de 1449, fecha ante quem 10 de febrero, Valencia. "Felipus Porta, pictor, civis Valencie, scienter et [gratis con]fiteor et in veritate recognosco vobis Felipo Cas(..), mercatori lombardo, residenti in dicta civitate, presenti, quod per manus honorabilis Iohannis Cavall, mercatoris, civis dicte civitatis, dedistis et solvistis michi, voluntati mee, [realiter nu]merando in presencia notari et testium infrascriptorum (...), quinquaginta quinque libris monete regalium Valencie [debi]tes per michi adsolvendum ex illis trecentis fllorinis] comunis aragonum, precio quorum ego pictavi et feci qu[em]dam retabulum in capella vocata Lombardorum, constructa in monasterio Sancti Francisci dicte civitatis, et in quibus fuistis condepnatus per sentenciam arbitrale latam magistros Iacobum Jacomart et Iohannem Rexach, pictores, in posse notari infrascripti continetur. Et quia rei veritas talis est; renuncians et cetera in quorum et cetera. Actum Valencie.Testes discretus Leonardus Gaya, notarius, et Petrus Dolesa, tintorerius, cives Valencie" Marti Doto, ARV, Protocolo 10.422. 
borar correctamente el definitivo catálogo de su producción pictórica. Porque, cabe advertir, además, que no se trata de una presencia de dos maestros por separado y sirviendo cada uno de ellos a dos causas, dos intereses o dos clientes diferentes como aconteció, por ejemplo, en Burjassot, entre el propio Reixach y el pintor Jaume Mateu ("En Jacme Matheu de part del senyor rey e En Johan Rexach, pintors, per part dels dits marmesors") ${ }^{14}$, sino que esta vez Jacomart y Reixach aparecen claramente como un único e indiviso dúo profesional perfectamente compacto y operativo: "magistros Iacobum Jacomart et Iohannem Rexach, pictores".

Hasta la aportación de esta importante noticia documental inédita, diversos historiadores del arte han emitido juicios y consideraciones de innegable riqueza que deben tenerse en cuenta. Mercedes Gómez Ferrer, por ejemplo, alude a este problema al afirmar que la entrada de Reixach en el panorama internacional coincide con el contrato de los dos más importantes retablos reales ${ }^{15}$, hecho que ha ocasionado que muchas de las pinturas hasta entonces atribuidas a Jacomart fueran, no siempre de modo documentado, restituidas a Reixach. De hecho, Fernando Benito, quizá un poco precipitadamente, abogó por reunir en la producción de este último pintor toda la obra considerada de Jacomart, ya que, según él, aunque de forma no suficientemente contrastada como hemos acabado de comprobar, no se conoce ninguna pieza suya conservada ${ }^{16}$. Esta tendencia es totalmente opuesta a la historiografía de inicios de siglo XX, que, como indicó Ferre ${ }^{17}$, atribuía a Jacomart las piezas de mayor calidad, por haber sido pintor del rey, antes que Reixach, dejando, para este último, aquellas más flojas y repetitivas; aludiendo a que el primero de ellos sería el creador de unos modelos, y el segundo, su continuador. Company ${ }^{18}$ opinó que no debía descartarse la posible colaboración entre Jacomart y Reixach en la realización de algunas obras atribuidas a ambos, y, por último, Mauro Natale y Gennaro Toscano ahondaron en esta hipótesis señalando algunas obras que pudieron ser realizadas en compañía ${ }^{19}$.

Otros investigadores como Mariano González Baldoví se han planteado cómo pudieron los dos mejores artistas del Reino trabajar juntos, afirmando que serían más bien rivales que lucharían por obtener el mayor número de encargos posibles ${ }^{20}$, algo que, como bien sabemos, a menudo era precisamente todo lo contrario, es decir, los grandes pintores se asociaron entre sí para de ese modo asegurarse y acaparar los mejores contratos del momento. Es lo que sucedió con las bien conocidas asociaciones entre Marzal de Sas y Pere Nicolau, Gonçal Peris ${ }^{21}$ y Guerau Gener, Bartolome Bermejo y los Osona, Fernando Yáñez de la Almedina y Fernando Llanos, Paolo da San Leocadio y Francesco Pagano, entre muchos otros ejemplos. De hecho, Montolío remarcó que fue Jacomart una suerte de catalizador del arte de Reixach, ya que su relación produjo "una seqüència artística reinterpretativa de les maneres i les formes del primer, garbillada pel cicle formatiu explícit de Reixac en l'àmbit internacional. Jacomart, com a millor pintor actiu en terres de la corona en temps d'Alfons el Magnànim, va influenciar de manera decisiva en les formes compositives de Reixac"22.

${ }^{4}$ Registre d'àpoques de la Batlia, ARV, núm. 46, f. 323.

15 Gómez-Ferrer, 2006: 77.

16 Benito Domenech, 2001: 31-45.

17 Ferre Puerto, 1996: 311-320.

18 Company, 2001: 332-339.

19 Natale, 2001: 19-45 y 323-328. Toscano, 2001: 329-331 y 340-344.

20 GoNZÁLEZ, 2003: 214-219.

21 A colación de este pintor, indicar a modo de inciso, que Reixach se formaría en su taller, pues en 1438 aparece documentado junto a Gonzalo Peris Sarrià y García Sarrià y unos años antes figuró como testimonio en un pago al pintor Jaume Mateu. No en vano, el 23 de septiembre de 1451 participó como testigo del testamento de Gonçal Peris Sarrià, uno de los máximos representantes del gótico internacional valenciano, estilo que Reixach superó en parte y adecuó a las nuevas modas y gusto de los comitentes.

22 Montolío, 2003: p. 224. 
En cualquier caso, tras la muerte de Jacomart en 1461, Reixach pasó a constituir la primera referencia del panorama pictórico valenciano del momento. Nombrado pintor real por Juan II en 1466, la multiplicación de su actividad profesional y su avanzada edad produjo que sus tipos iconográficos y estilísticos se estandarizaran, puede que también fruto de la comprensible mano de obra del taller. Quizá a la franja cronológica de este momento pertenecería el citado Retablo de San Lorenzo y San Pedro de Verona de Catí (1461), que como se ha dicho contrató Jacomart en 1460, y pudo ser conjuntamente realizado por Jacomart y Reixach, además de con el concurso de otros miembros del taller de ambos, o el Retablo de San Esteban para la iglesia homónima de Valencia (1462). También de la década de los sesenta son el Retablo de la Capilla Real del Convento de Santo Domingo (1463) o las puertas del órgano de la Catedral de Valencia (1465).

A nuestro juicio, es en este periodo álgido donde deberíamos incluir la obra objeto de nuestro estudio, es decir, en ese momento de efervescencia productiva, donde continúa desarrollándose esa simbiosis entre arte tardogótico e hispanoflamenco, unido a una cierta renovación clásica ejemplificada por el equilibrio compositivo, si bien el uso de la perspectiva aún dista de la perfección del Quattrocento italiano.

Su fama se hizo extensible a todos los lugares de la antigua Corona de Aragón, así pues, conservamos encargos como el Retablo de Santa Ursula del Museu Nacional d'Art de Catalunya (1468), procedente de la localidad de Cubells (Lleida), pero elaborado para el monasterio cisterciense de Poblet (Tarragona); o también la tabla de la Visitación de Morella, Castellón.

Los últimos momentos de actividad pictórica del taller de Reixach son apreciables, por ejemplo, en las tablas para Museros (1470), para la cofradía de San Jaime de Valencia (1470-1472), además del encargo particular del mercader Joan Sánchez en 1484. En este año aparecerá relacionado con Pere Cabanes, según nos demuestra un pleito mantenido con el vecino grupo de pintores de cajas y arquibancos ${ }^{23}$. La mayor parte de las últimas obras citadas suponen una estandarización de un arte novedoso que supuso una transición hacia el primer Renacimiento, pero sin abandonar el talante marcadamente gótico de su factura. Sus tipos iconográficos así como sus coloraciones sirvieron de modelo a pintores posteriores (sobre todo al extenso círculo del Maestro de Perea) y constituyeron el culmen al excelente arte medieval valenciano. Como colofón a su conocida actividad pictórica podemos marcar una de sus últimas obras documentadas, el Retablo Mayor del convento de Santa María Magdalena de Valencia (1486), pocos años antes de su muerte, aproximadamente en 1490 , que pudo ejemplificar a la perfección su estilo sobrio, comedido, detallista y mesurado que tanto gustó a los comitentes y público valenciano de la segunda mitad del siglo XV, con formas y colorido vivaz e indudable fuerza expresiva.

\section{BIBLIOGRAFIA}

Baticle, Jeannine; Marinas, Cristina, La Galerie Spagnole de Louis-Philippe au Louvre, 1838-1848, París, 1981.

Benito Doménech, Fernando, “Evocaciones flamencas en los primitivos valencianos”, en Benito Doménech, Fernando (comisario), La clave flamenca en los primitivos valencianos, cat. exp., Valencia, Generalitat Valenciana, 2001, pp. 23-62.

Cerveró, Luis, "Pintores Valentinos. Su cronología y documentación", en Archivo de Arte Valenciano, 37, 1966, pp. 19-30.

Company, Ximo; Puig, Isidro, "Flagelación. Joan Reixac", en Company, Ximo; Pons, Vicent; Aliaga, Joan, La Llum de les Imatges, Lux Mundi, cat. exp., Xàtiva, Generalitat Valenciana y Fundació La Llum de les Imatges, 2007, núm. 99, pp. 330-333.

23 COMPANy, 2001: 333-337, basándose en el documento publicado por SANCHIS SivERA, 1930: 207-208. 
Company, Ximo, “Jaume Baço, llamado Jacomart y Joan Reixach: Tríptico de la Virgen con el Niño, ángeles, la reina María de Castilla como donante, san Miguel y san Jerónimo, con las puertas abiertas; arcángel Gabriel y la Virgen anunciada, con las puertas cerradas, c.1448-1458”, en Natale, Mauro (comisario), El Renacimiento Mediterráneo, cat. exp., Madrid, 2001, pp. 332-339.

Ferre, Josep, "Joan Reixac, autor de dues obres del cercle Jacomart-Reixac", en Actes del Primer Congrés d'Estudis de la Vall d'Albaida, Valencia, Diputació de València, 1997, pp. 311-320.

Ferre, Josep, "Jacomart, lo feel pintor d'Alfons el Magnànim: Puntualitzacions a l'obra valenciana", en D’Agostino, Guido (ed.), XVI Congresso Internazionale di Storia della Corona d'Aragona, Nápoles, Paparo Edizioni, 2000, vol. II, pp. 1681-1686.

Gómez-Ferrer, Mercedes, "Un nuevo contrato de Jacomart: El retablo de la Iglesia Parroquial de Museros", en Archivo de Arte Valenciano, 75, 1994, pp. 20-24.

Gómez-Ferrer, Mercedes, "Jacomart: revisión de un problema historiográfico", en Hernández, Lorenzo (coord.), De pintura valenciana (1400-1600), Alicante, Instituto Alicantino de Cultura Juan Gil-Albert, 2006, pp. 71-100.

Gómez-Ferrer, Mercedes; Corbalán de Celis, Juan, "El retablo de la parroquial de Museros, obra de Joan Reixach", en Archivo de Arte Valenciano, 84, 2003, pp. 19-24.

González, Mariano, “Joan Reixac o Pere Joan Reixac: Santa Ana, la Virgen y el Niño”, en Ruiz, Francesc (comisario), La pintura gòtica hispanoflamenca. Bartolomé Bermejo i la seva època, cat. exp., Barcelona, MNAC y Museo de Bellas Artes de Bilbao, 2003, pp. 214-219.

Madurell, José María, "El arte en la comarca alta de Urgel", Anales y Boletín de los Museos de Arte de Barcelona, vol. III, núm. 4, 1945, pp. 259-340.

Montolío, David, "Biografia de Joan Reixac", en Ruiz, Francesc (comisario), La pintura gòtica hispanoflamenca... cat. exp., Barcelona, MNAC y Museo de Bellas Artes de Bilbao, 2003, pp. 241 y 216.

Natale, Mauro, "El Mediterráneo que nos une" y "Atribuido a Jacomart: Virgen Anunciada, c. 1450", en Natale, Mauro (comisario), El Renacimiento Mediterráneo, cat. exp., Madrid, 2001, pp. 19-45 y 323-328 respectivamente.

Saralegui, Leandro de, "De pintura valenciana medieval. En torno al binomio Jacomart-Reixac", en Archivo de Arte Valenciano, 36, 1962, pp. 5-12.

Serrano, Manuel, "Documentos relativos a la pintura de Aragón durante los siglos XIV y XV", en Revista de Archivos, Bibliotecas y Museos, 34, 1916, pp. 455-485.

Terés, Maria Rosa, "Llorenç Reixac, escultor de la catedral de Barcelona”, Lambard, vol. III, 1987, pp. 171185.

Tormo, Elías, “Los pintores cuatrocentistas: Juan Rexach”, Cultura Española, XI, 1908 pp. 1064-1079.

Tormo, Elías, Jacomart y el arte hispano-flamenco cuatrocentista, Madrid, Centro de Estudios Históricos, 1913.

Toscano, Gennaro, “Taller de Jacomart-Reixac: Verónica de la Virgen y de Cristo, c. 1450” y "Taller de Jacomart-Reixach: Santa Elena y San Sebastián, c. 1460", en Natale, Mauro (comisario), El Renacimiento Mediterráneo, cat. exp., Madrid, 2001, pp. 329-331 y 340-344 respectivamente.

\section{Fuentes documentales}

Archivo de la Catedral de Valencia (ACV), protocol núm. 3.680.

Archivo de Protocolos del Patriarca de Valencia (APPV), Protocols de Bertomeu Tovià, núm. 25.047.

Arxiu del Regne de València (ARV), Mestre Racional, núm. 55.

Arxiu del Regne de València (ARV), Registre d'àpoques de la Batlia, núm. 46.

Arxiu del Regne de València (ARV), Marti Doto, Protocol núm. 10.422.

Arch. esp. arte, LXXXV, 340, OCTUBRE-DICIEMBRE 2012, 351-387, ISSN: 0004-0428 